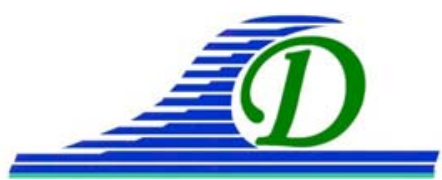

XIII ${ }^{\text {èmes }}$ Journées Nationales Génie Côtier - Génie Civil

Dunkerque, 2-4 juillet 2014

DOI:10.5150/jngcgc.2014.060 @ Editions Paralia CFL

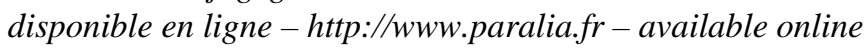

\title{
Modèles numériques de bathymétrie pour la prévision hydrodynamique du dispositif vigilance vagues-submersions
}

\author{
Laurie BISCARA ${ }^{1}$, Thierry SCHMITT ${ }^{1}$, \\ Stéphanie CORREARD ${ }^{2}$, Ronan CREACH ${ }^{1}$
}

1. SHOM, 13 rue du Chatellier, 29200 Brest, France.

laurie.biscara@shom.fr

2. SHOM, Campus de Météo France, 42 avenue Gaspard Coriolis, 31400 Toulouse,

France.

\section{Résumé :}

Suite aux évènements catastrophiques de ces dernières années (tempête Johanna de 2008 et Xynthia de février 2010 notamment), la Direction Générale de la Prévention des Risques mène une démarche au profit de la sécurité des personnes dans les zones exposées aux phénomènes brutaux de submersions rapides dont, notamment, celles d'origine marine. Afin de produire des prévisions aussi précises que possible des hauteurs d'eau et d'états de mer à la côte dans le cadre du projet HOMONIM, de nouveaux Modèles Numériques de Terrain (MNT) sont réalisés pour la métropole et implémentés dans les modèles hydrodynamiques. Trois MNT d'emprise et de résolution différentes couvrant la façade Atlantique ont été construits jusqu'ici: 1) le MNT régional à $500 \mathrm{~m}$, allant de la Mer du Nord jusqu'à Gibraltar, 2) le MNT de façade à 100 $\mathrm{m}$, le long de la côte métropolitaine et 3) le MNT côtier à $20 \mathrm{~m}$ sur la zone à enjeux Xynthia (pertuis charentais). La construction de ces MNT s'est appuyée majoritairement sur les données extraites de la Base de Données Bathymétriques du SHOM. Les régions présentant des lacunes de données ont été comblées à la côte à l'aide des sondes non numérisées mais existantes sur les cartes marines et au large avec des MNT globaux à plus basse résolution. Ce travail s'attache plus particulièrement à détailler la méthodologie de production du MNT de façade ainsi que les solutions retenues.

Mots-clés : Bathymétrie, MNT, HOMONIM, Xynthia, Interpolation, Conversion altimétrique, Modèles hydrodynamiques, Vigilance vague-submersion.

\section{Introduction}

\subsection{Généralités}

Les plans d'action de la puissance publique en matière de prévention des risques côtiers sont mis en œuvre dans différents dispositifs dont fait partie le plan national "submersions rapides" (PSR). L'identification et la cartographie précise des zones à risques d'inondation, y compris par submersion constituent ainsi des objectifs majeurs afin de pouvoir établir des dispositifs de prévision prévus par le PSR. Pour cela, il s'agit 
essentiellement de pouvoir produire des prévisions aussi précises que possible des hauteurs d'eau et d'états de mer à la côte pour améliorer la pertinence de la Vigilance Vague-Submersion (VVS). Coordonné par Météo France et le SHOM, le projet HOMONIM (Historique, Observation, Modélisation des Niveaux Marins) s’inscrit dans le cadre de la VVS et a pour objectif d'améliorer les prévisions de surcote et de hauteur significative des vagues. Dans le cadre de ce projet, des MNT sont réalisés pour la métropole et implémentés dans les modèles hydrodynamiques.

\subsection{Définition du besoin en MNT}

Le système de modélisation côtier du projet utilise une série de modèles hydrodynamiques emboîtés allant du large à la côte. Trois gammes de MNT bathymétriques sont ainsi définies (Fig. 1 \& Fig.2) :

- Modèle régional :

Il s'agit de fournir une condition aux limites pour les modèles de façade. Le modèle régional couvre une partie d'un océan et s'étend au-delà du talus. La résolution visée en bathymétrie atteint $500 \mathrm{~m}$.

- Modèle de façade :

Il peut correspondre au modèle final pour certaines applications ou servir de conditions aux limites pour le modèle côtier. Son emprise englobe tout ou partie d'une façade maritime nationale. Les résolutions des MNT atteignent $100 \mathrm{~m}$.

- Modèle côtier :

Il correspond au modèle final sur lequel les applications sont visées. Son emprise est typiquement de $10 \mathrm{~km}$ à $1 \mathrm{~km}$. En zone littorale, les phénomènes physiques modélisés sont très sensibles aux détails de la bathymétrie locale. Les résolutions nécessaires peuvent atteindre moins de $10 \mathrm{~m}$ à proximité des zones à enjeux et sont le plus souvent comprises entre 10 et $50 \mathrm{~m}$. Il peut bénéficier de données LIDAR (Litto3D ${ }^{\circledR}$ ) lorsque celles-ci sont disponibles, ces dernières étant par ailleurs utiles pour modéliser les inondations.

\subsection{Zone d'intérêt}

Cet article présente trois MNT d'emprise et de résolution différentes couvrant la façade Atlantique (Fig. 2A) : (1) le MNT régional à 500 m, allant de la Mer du Nord jusqu'à Gibraltar; (2) le MNT de façade à 100 m, le long de la côte métropolitaine et (3) le MNT côtier à 20 m sur la zone à enjeux Xynthia (pertuis charentais). 


\section{XIII ${ }^{\text {èmes }}$ Journées Nationales Génie Côtier - Génie Civil \\ Dunkerque, 2-4 juillet 2014}

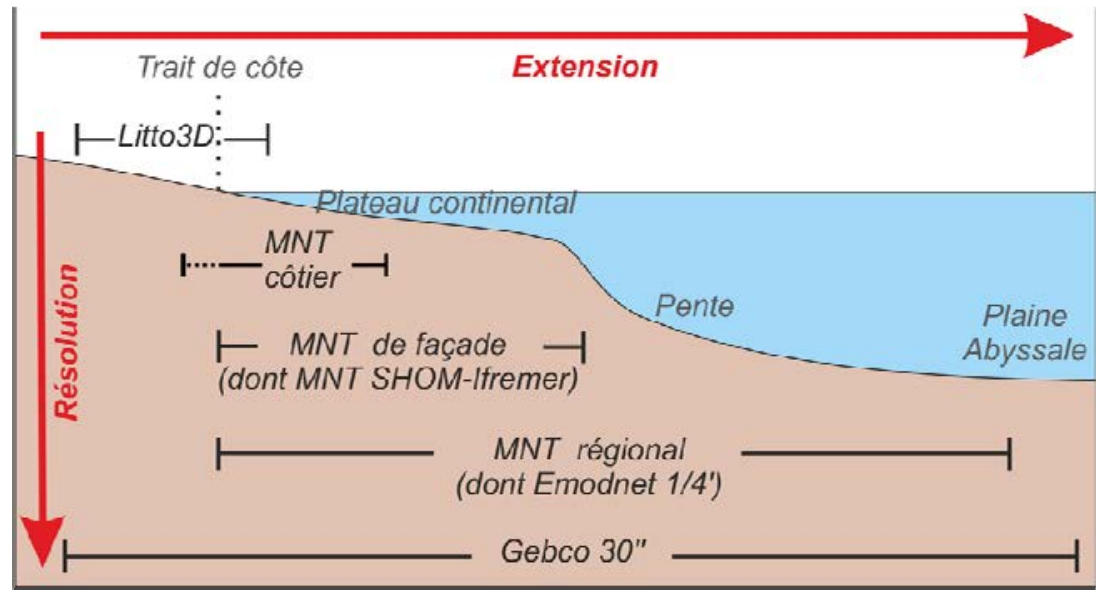

Figure 1. Gamme de MNT disponibles en fonction de leur extension et de leur résolution (adapté d'après HEAKINS et al., 2013).

\section{Méthodologie}

Seule la méthodologie employée pour construire le MNT de façade est présentée dans cette section.

\subsection{Origine des données}

\subsubsection{Trait de côte}

Le trait de côte utilisé pour délimiter le MNT de façade est Histolitt ${ }^{\circledR}$ pour la France et GSHHS ("A Global Self-consistent, Hierarchical, High-resolution Geography Database", produit par la NOAA (http://www.ngdc.noaa.gov/mgg/shorelines/gshhs.html) pour les pays limitrophes. Le trait de côte Histolitt correspond à la limite de la plus haute mer astronomique (PHMA) tandis que le GSHHS correspond à la limite de la "Mean High Water" (MHW).

\subsubsection{Bathymétrie}

La création du MNT s'est appuyée sur les données issues de la Base de Données Bathymétrique du SHOM (BDBS). Les secteurs restant sans couverture bathymétrique sont finalement comblés à l'aide de sondes de levés SHOM non numérisés issus des ENC ("Electronic Navigational Charts") à la côte puis par le MNT de plus basse résolution (500 m).

- Les données SHOM :

Les sondes sont extraites de la BDBS. Les levés contenus en base ont été acquis sur une large période de temps, les levés les plus anciens datant du $19^{\text {ème }}$ siècle. Bien que dans les zones à fond évolutif ces levés ne soient plus à jour de la connaissance, ils restent néanmoins indispensables dans certaines régions lorsqu'ils constituent l'unique source de données bathymétriques. Les méthodes d'acquisition, de 


\section{Thème 3 - Instrumentation, mesures, imagerie et télédétection}

traitement ainsi que les incertitudes horizontales et verticales des sondes sont conformes aux normes (OHI, 2008, S-44 par exemple) en vigueur au moment de l'acquisition de celles-ci. De manière générale, les sondes sont donc issues de capteurs hétérogènes et acquises à des époques différentes mais toutes réduites de la marée et rapportées à un zéro hydrographique de la zone de marée correspondant au lieu du levé et exprimées dans le système géodésique WGS84 (ou assimilé RGF93 le cas échéant).

- Les données des ENC :

En plus des données de la BDBS, des sondes issues des ENC ont été ajoutées dans les zones côtières où des lacunes de données numériques existent. Les sondes présentes dans les ENC n'apparaissent pas nécessairement dans la BDBS car elles peuvent être issues de levés extérieurs ou d'anciens levés SHOM non numérisés. Plusieurs dizaines de milliers de sondes et de relevés topographiques sur l'estran ont ainsi été ajoutés à l'extraction initiale sur la côte Aquitaine, aux abords de Dunkerque et en baie du Mont St-Michel, respectivement.

- MNT Global (Emodnet) :

Les secteurs au large, non couverts par les données précédentes sont comblés à l'aide du MNT Emodnet, en diffusion libre sur le portail Emodnet-Hydrography (http://www.emodnet-hydrography.eu). La grille Emodnet, diffusée à une résolution de 0.25 ' couvre en partie l'Atlantique Nord-Est et la Méditerranée. Elle est issue d'un assemblage hétérogène de données dont le référencement vertical est assumé comme la PBMA (EUROPEAN MARINE OBSERVATION AND NETWORK, 2011). Malgré sa bonne résolution à l'échelle régionale, la grille Emodnet souffre d'artéfacts liés à l'exploitation de différentes sources.

\subsubsection{Grilles de conversion altimétrique}

Le MNT fourni est référencé au niveau moyen (NM) pour les besoins de modélisation. La grille bathymétrique est ainsi référencée à la plus basse mer astronomique (PBMA), à laquelle s'associe une grille de conversion altimétrique PBMA-NM d'extension et de résolution similaires. Ces corrections s'appliquent pour des profondeurs d'eau inférieures à 200 m. Au-delà, la référence verticale peut être assimilée au NM. Dans un premier temps les données SHOM sont recalées à la PBMA (Fig. 2C). En effet, la référence verticale des données SHOM est le zéro hydrographique $(\mathrm{ZH})$. Bien qu'il soit par définition voisin de la PBMA, les écarts entre ces deux niveaux peuvent être significatifs et atteindre jusqu’à $50 \mathrm{~cm}$. La conversion altimétrique est réalisée à l'aide du produit Bathyelli, qui cote les différentes surfaces de références par rapport à l'ellipsoïde GRS80 de l'IAG. En raison de l'extension de la grille Bathyelli limitée à la façade métropolitaine, le modèle Marmonde a ensuite été utilisé pour créer la grille de conversion altimétrique PBMA-NM. Un modèle de marée a d’abord été généré dont a été extraite la composante de NM par rapport à la PBMA (Fig. 2E). 


\section{XIII ${ }^{\text {èmes }}$ Journées Nationales Génie Côtier - Génie Civil \\ Dunkerque, 2-4 juillet 2014}

2.2 Processus d'élaboration des modèles numériques de terrain

Les données ont été traitées à l'aide de GMT (http://gmt.soest.hawaii.edu) (WESSEL \& SMITH, 2013 ; version 4.5.2) sous environnement Linux.

\subsubsection{Assemblage et traitement des données issues de la BDBS}

Près de 4700 lots de données ont été extraits sur la zone d'étude. Les données ont ensuite été décimées ( 1 sonde conservée tous les $10 \mathrm{~m}$ ), assemblées puis moyennées au pas de sortie (blockmedian) afin de préparer les données à l’interpolation.

\subsubsection{Evaluation de la couverture des données}

Afin d'évaluer le taux de couverture des données SHOM, des interpolations par voisin naturel avec différents paramètres de recherche sont réalisées (nearneighbor, Fig. 2B). Cette méthode permet : 1) d'évaluer le taux de couverture en fonction des paramètres d'interpolation, 2) de repérer les lacunes de données à la côte, qui sont ensuite comblées à l'aide des ENC et 3) d'utiliser l'emprise des données interpolées comme masque pour la sélection des données complémentaires.

\subsubsection{Comblement du MNT}

Dans la mesure du possible, la source SHOM a été exploitée en priorité sur la bande 0$200 \mathrm{~m}$, afin de limiter les discontinuités à la jonction de différentes sources. Dans le cas du MNT de façade, l'interpolation seule des données issues de la BDBS n'était pas suffisante pour permettre une couverture continue sur le plateau du Golfe de Gascogne (Fig. 2B). En raison des artéfacts observés sur la grille Emodnet et dans un souci d'homogénéité, il a été décidé de combler les lacunes à l'aide du MNT 500 m rééchantillonné (Fig. 2D). La grille Emodnet est quant à elle exploitée sur la partie Nord de la Manche, où elle présente une bonne cohérence morphologique (Fig. 2D).

\subsubsection{Interpolation des données}

Une fois la sélection des différentes sources effectuée, les données sont finalement assemblées pour être interpolées. Le MNT est généré en utilisant une fonction spline régularisée avec tension (surface). Cette méthodologie permet de limiter les artéfacts à la jonction entre les différentes sources (Fig. 2F). 

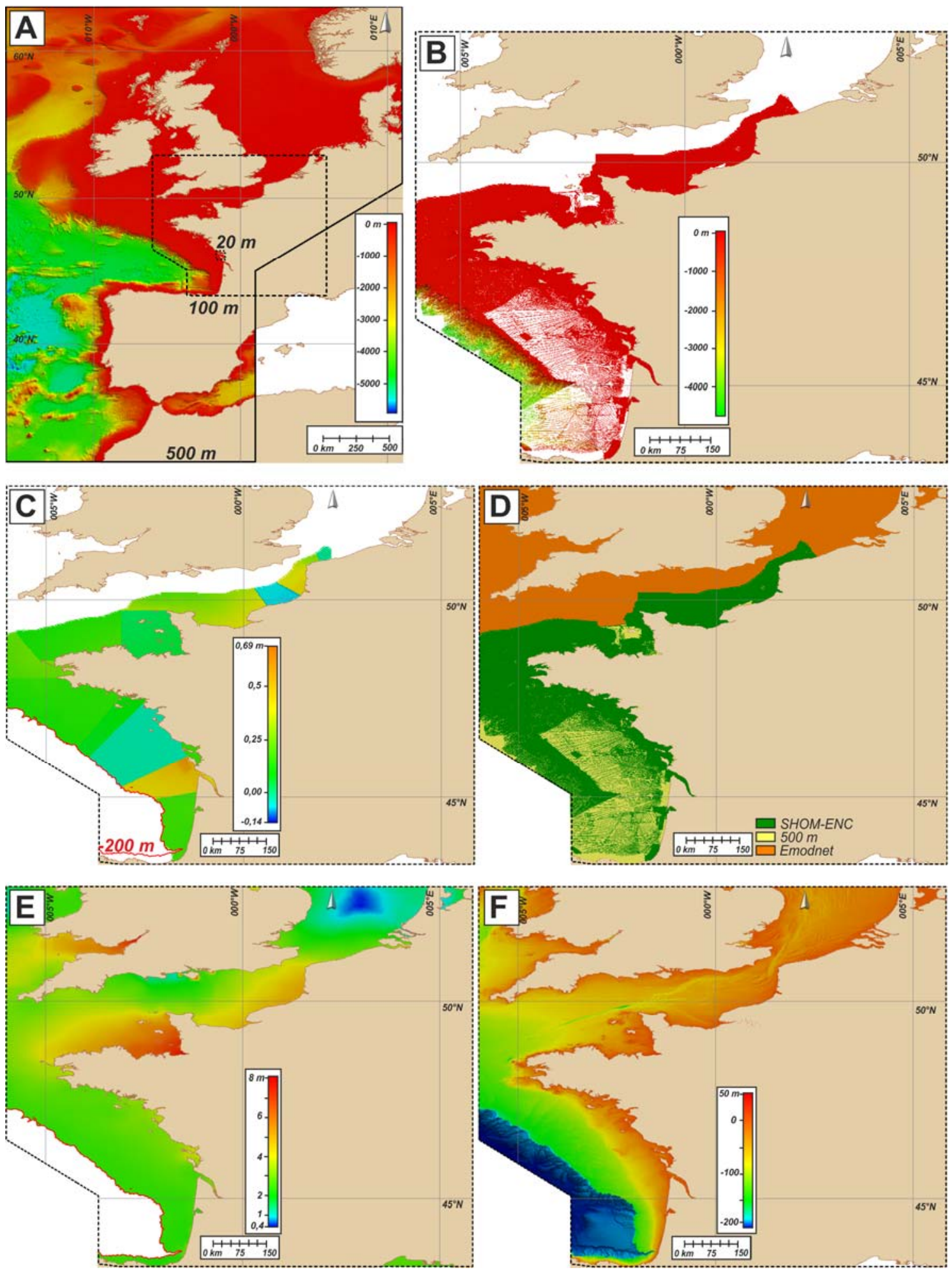

Figure 2. Etapes de production du 100 m Homonim. A: Emprise des différentes MNT; B: taux de couverture des données SHOM; C: Grille de conversion altimétrique ZHPBMA Bathyelli; D: Autres sources exploitées pour le comblement du MNT; E: Grille de conversion altimétrique PBMA-NM Marmonde; F: MNT final. 


\section{XIII ${ }^{\text {èmes }}$ Journées Nationales Génie Côtier - Génie Civil \\ Dunkerque, 2-4 juillet 2014}

\section{Résultats et discussion}

La construction de ce modèle de façade a bénéficié des réflexions menées sur la production de MNT au SHOM, notamment dans le cadre du projet commun SHOMIfremer (SCHMITT et al., 2013). Ainsi une grille représentant la ou les sources de données utilisées pour la création de chaque nœud a pu être livrée. Elle apporte de manière indirecte des éléments concernant la qualité de l'information produite. L’étude initiale du taux de couverture des données a également permis d'exploiter au mieux les informations des ENC pour combler les lacunes de données en sortie d'extraction de la BDBS. Bien que la densité des données soit faible, cet ajout aide à contraindre l'interpolation et à limiter l'assemblage de données de différentes sources en milieu côtier. Enfin l'utilisation d'un interpolateur unique sur l'ensemble des données a limité les discontinuités morphologiques à la jonction entre les différentes sources.

Le MNT 100 m obtenu couvre une gamme de profondeur allant du domaine côtier jusqu'à environ $4500 \mathrm{~m}$ de profondeur et présente une bonne cohérence globale. En domaine côtier se pose néanmoins la question de la composante temporelle de la bathymétrie utilisée, notamment dans les secteurs à forte dynamique sédimentaire. La figure 3 présente par exemple la morphologie obtenue au niveau des pertuis charentais pour le MNT de façade et côtier. On constate sur le MNT $100 \mathrm{~m}$ la représentation inexacte de deux chenaux au niveau du pertuis de Maumusson alors que l'ENC (produit le plus à jour de la connaissance) n'en représente qu'un seul dans ce secteur. Les levés acquis dans cette zone respectivement ont ainsi cartographié différentes positions du chenal au cours du temps. Cette observation est confirmée par les travaux de BERTIN et al. (2004) montrant le basculement du chenal vers le Sud entre 1824 et 2001. Au contraire, sur le MNT côtier, la sélection unique des levés les plus récents dans ces zones mobiles a permis de représenter le plus fidèlement possible la morphologie du fond par rapport à l'actuel.

L'évolution naturelle des fonds (érosion des fonds, apports sédimentaires par les fleuves) sur des échelles de temps parfois très rapides révèle qu’il est impératif de réactualiser l'état de la connaissance bathymétrique dans les zones à forte dynamique sédimentaire. Il apparaît également essentiel de sélectionner l'information la plus pertinente dans le cas de fonds bathymétriques anciens. La sélection manuelle des levés et leur comparaison aux cartes de navigation ou à des images aériennes, permettra de construire un produit le plus à jour de la connaissance actuelle. 


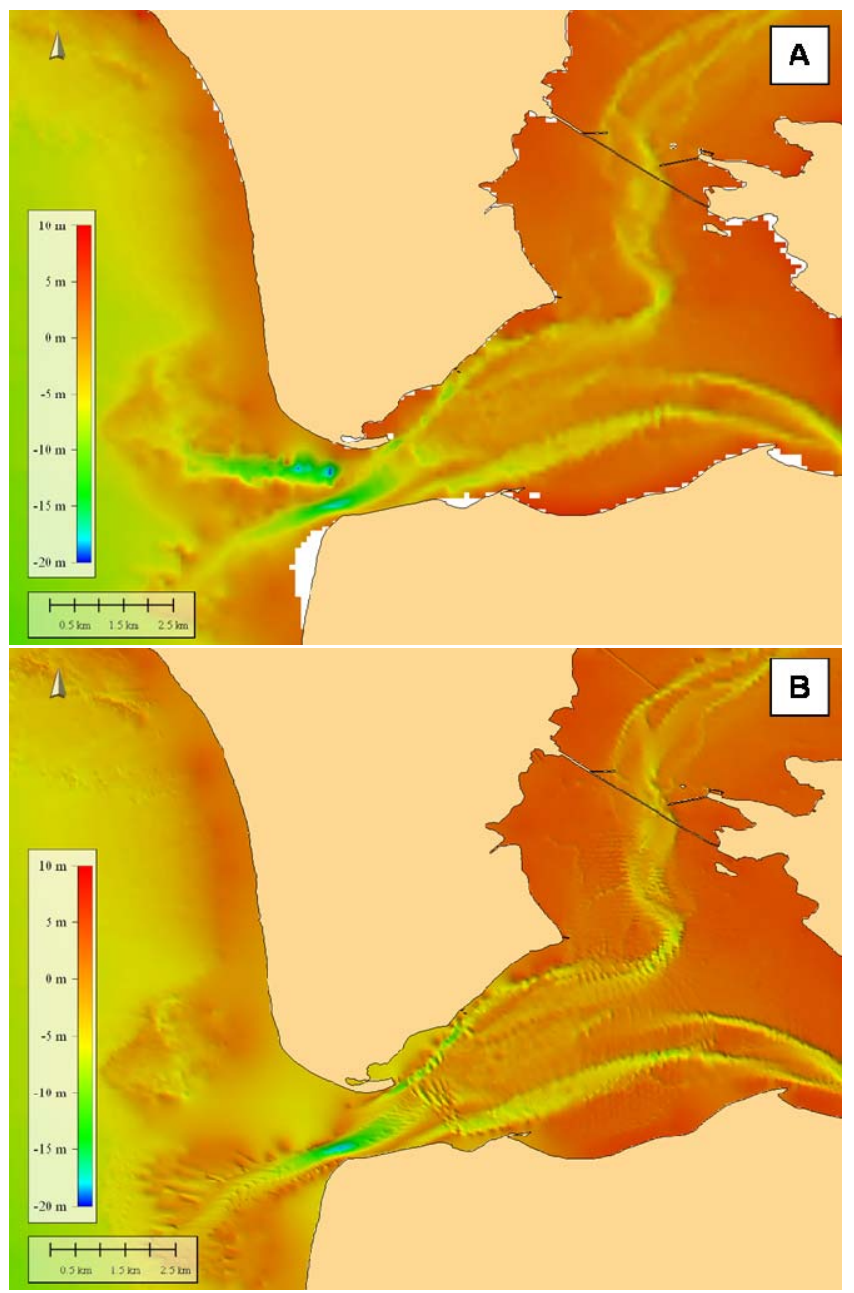

Figure 3. Comparaison de la morphologie du MNT $100 \mathrm{~m}$ (A) et $20 \mathrm{~m}$ (B) au niveau des pertuis Charentais. Deux chenaux sont mis en évidence sur le MNT 100 m, suggérant la cartographie de la position du pertuis à différentes périodes.

\section{Conclusion}

Inscrit dans le cadre de la VVS, le projet HOMONIM a pour but d'améliorer les prévisions de surcote et de hauteur significative des vagues en s'appuyant sur de nouveaux MNT implémentés dans les systèmes de modélisation hydrodynamique. Dans la continuité des produits déjà réalisés en Atlantique Nord-Est, une maquette similaire est en cours de préparation sur la Méditerranée. Des réflexions concernant le processus de construction de MNT de grande emprise restent à poursuivre afin d'aboutir à une chaîne de production systématisée, permettant notamment la correction de certains défauts ponctuels et la tenue à jour du MNT par réédition suite à la réalisation de nouveaux levés, de travaux de numérisation ou de nouveaux MNT globaux. La sélection des levés les plus récents en cas de superposition de travaux hydrographiques d’âges différents (processus déjà réalisé pour la construction du MNT côtier), se doit à 


\section{XIII ${ }^{\text {èmes }}$ Journées Nationales Génie Côtier - Génie Civil \\ Dunkerque, 2-4 juillet 2014}

terme d'être étendue sur l'ensemble de la façade maritime française. A la côte, le MNT Xynthia devrait permettre d'évaluer l'apport de la très haute résolution sur les prévisions de hauteur d'eau et d'état de mer. Néanmoins, le manque actuel de ce type de MNT concerne le continuum terre-mer. Les données Litto3D ${ }^{\circledR}$, particulièrement bien adaptées à ce genre de besoin se doivent à terme d'être intégrées dans les MNT côtiers. L'apport des grilles de conversion altimétriques issues du projet Bathyelli (PINEAUGUILLOU \& DORST, 2011) permettant la maîtrise des références verticales facilitera à l’avenir l'intégration des données Litto3D ${ }^{\circledR}$ référencés à l’IGN69.

\section{Références bibliographiques}

EUROPEAN MARINE OBSERVATION AND NETWORK (2011). Guidelines for metadata, data and DTM QA/QC, 21 p. http://www.emodnethydrography.eu/documents/emodnet_hydrography/html_page/qa_qc_dtm_specifications_040211-v4.doc

BERTIN X., CHAUMILLON E., WEBER N., TESSON M., (2004). Morphological evolution and time-varying bedrock control of main channel at a mixed energy tidal inlet: Maumusson Inlet, France. Marine Geology, Vol. 204(1-2), pp 187-202. http://dx.doi.org/10.1016/S0025-3227(03)00353-0

HEAKINS B., DANIELSON J., BROCK J., SUTHERLAND M., MCLEAN S. (2013). Creating a Framework for Integrated U.S. Digital Elevation Models. 2013 GSA Annual Meeting, Denver, 45(7), 649 p. https://gsa.confex.com/gsa/2013AM/finalprogram/abstract_229169.htm OHI -Organisation Hydrographique Internationale- (2008). S44 - Normes OHI pour les levés hydrographiques. $5^{\text {ème }}$ édition, Monaco. http://www.ho.int/iho_pubs/standard//s-44_5E.pdf PINEAU-GUILLOU L., DORST L. (2011). Creation of Vertical Reference Surfaces at Sea using Altimetry and GPS. Annales Hydrographiques, Vol. 777(7), pp 1-7 http://www.shom.fr/fileadmin/SHOM/PDF/02-Produits/Annales hydrographiques/Annales/AH777/10-ref-surface.pdf

SCHMITT T., LOUBRIEU B., AVISSE L., BISCARA L. (2013). Rapport d'évaluation du Modèle Numérique de Terrain conjoint SHOM IFREMER $100 \mathrm{~m}$ (Version 0). Rapport interne SHOM et Ifremer, $67 \mathrm{p}$.

WESSEL P., SMITH W. H. F. (2013). The Generic Mapping Tools Version 4.5.11 Technical Reference and Cookbook. 250 p. http://gmt.soest.hawaii.edu/gmt4/gmt/pdf/GMT_Docs.pdf 
Thème 3 - Instrumentation, mesures, imagerie et télédétection 